\title{
EXISTENCE OF EXTREMAL METRICS ON ALMOST HOMOGENEOUS MANIFOLDS OF COHOMOGENEITY ONE*
}

\author{
DANIEL GUAN ${ }^{\dagger}$ AND XIUXIONG CHEN ${ }^{\ddagger}$
}

Dedicated to Professor L. K. Hua, who was the teacher of Professor J. Q. Zhong. Zhong was the teacher of Guan in China and led him into this topic. Guan still have copies of papers of Calabi and Futaki which Zhong showed him in 1986.

1. Introduction. It is well-known that every Kähler class of a compact Kähler homogeneous manifold admits a Kähler metric with constant scalar curvature. We may ask following question:

Can we find any special metric on a compact almost homogeneous manifold of cohomogeneity One?

We have found this kind of metrics for a compact almost homogeneous manifold with two ends in $[\mathrm{Gu} 1,2,3]$, i.e., there are both extremal metrics and quasi-Einstein metrics in each Kähler class ${ }^{1}$. Then we may ask if the same is true for all the compact almost homogeneous manifolds of cohomogeneity one, which are described in both $[\mathrm{Ah}]$ and $[\mathrm{HS}]$.

If the algebraic part of the automorphism group is not semisimple, the manifold has a structure of a completion of a $\mathbf{C}^{*}$ bundle over a compact homogeneous Kähler manifold (see Theorem 1). According to the results in [Gu1,2,3], there always exists an extremal metric (resp. a quasi-Einstein metric) in each Kähler class. Hence, the problem of finding extremal metrics (resp. quasi-Einstein metrics) is reduced to the situation in which the algebraic part of the automorphism group is semisimple. By abusing the language, we call this case the semisimple case.

The semisimple case is also very interesting since any invariant Kähler metric is not a very good metric in the real Riemannian geometry. It is not a metric invariant under a maximal compact Lie group. For example, if a general hypersurface orbit $K / L$ has a right circle action $S_{1}$ (there is one always in our case), then the whole manifold has a circle action introduced by this action. By averaging method, we obtain a Riemannian metric which is invariant under $K \times S_{1}$. However, the new metric can not be Kähler since otherwise the automorphism group of $M$ is bigger than $K_{\mathbf{C}} \times \mathbf{C}^{*}$ - a contradiction to our assumption. So some methods in the real Riemannian manifold of cohomogeneity one, e.g., the method of circle action, do not work for any one here.

\footnotetext{
${ }^{*}$ Received April 3, 1999; accepted for publication April 13, 1999.

†Dept. of Math., Univ. of Cal., Riverside, CA 92521, U.S.A. (zguan@math.ucr.edu). Supported by NSF Postdoctoral Fellowship DMS-9627434.

$\ddagger$ Department of Mathematics, Princeton University, Princeton, NJ 08544, U.S.A. Partially Supported by NSF Postdoctoral Fellowship.

${ }^{1}$ In our preparing of [Gu6], we find that the proof of the existence for Kähler metrics of constant scalar curvature on almost homogeneous manifolds with two ends and reductive automorphism group in [Hw1] is not complete. For example, the Theorem 3 there follows from his Theorem 2, but in the case of Theorem 2 the base manifold is a product of manifolds with $b_{2}=1$. Therefore, the proof of his Theorem 4 can come from neither his Theorem 3 nor his Theorem 2. Neither the proof of existence of extremal metrics on almost homogeneous manifolds of cohomogeneity one is true-as we see in this paper. Also, there should be nonnegative conditions of the Ricci curvatures in the Theorem 2 and Corollary 2 in [Gu3], the "B" in the (3) of [Gu3 p.374] should be "B and r". Although the proof there actually works for the case in which the traces $r^{j}$ of $r$ on the eigenspaces of $B$ are constants with at most one negative $r^{j}$ which corresponds to an eigenvalue $b_{j}$ of $B$ such that there is no other eigenvalue of $B$ between $b_{j}$ and zero, e.g., $B=k g_{t}, g_{t}$ have constant scalar curvatures.
} 
In the semisimple case we notice that all the extremal metrics (resp. the quasiEinstein metrics) are metrics with constant scalar curvatures. This is our initial interest since all the manifolds with constant scalar. curvatures have reductive automorphism groups. In the semisimple case, the Futaki invariants are automatically zero (see Theorem 2). Also, if there is an extremal metric on the algebraic part of the manifold, by producting with the standard metric on the Albanese torus we obtain an extremal metric on a covering of the whole manifold.

In general, the metrics of these manifolds are not easy to deal with. Therefore, we have to look at some examples first. We did not obtain any simple nontrivial example until we finished [Gu4].

Motivated with the Hilbert scheme construction in [Gu4], we consider the manifold (see [Ch] for the notations) $M_{n}=Z_{1,2}\left(\mathbf{C} P^{n}\right)$ 's constructed by blowing up the diagonal of the product of the two copies of a complex projective space and the manifold $N_{n}=\operatorname{Hilb}^{2}\left(\mathbf{C} P^{n}\right)$ 's constructed from $M_{n}$ 's by modulo the symmetry group.

The manifold $M_{n}$ 's are Fano, while $N_{n}$ 's are not Fano but almost Fano in the sense that the anti-canonical line bundles are nef.

The calculation is much more complicate than that in [Gu2,3]. In this paper we focus on Fano and the Ricci class case of $M_{n}$ 's, i.e., the Kähler-Einstein cases. We will prove the existence of the Kähler-Einstein metrics on $M_{n}$ 's.

It seems to us that the difficulties in $[\mathrm{Gu} 2,3]$ are concentrated at the positivity of solutions of certain first order equations. But in this paper the difficulty is more concentrated at the existence of the solutions of some second order equations. Once we have solutions, we can obtain the positivity. It also seems to us that the existing theories of Sakane \& Koiso, Siu, Tian, Nadel (see [SK1, Siu, Ti, Nd1,2]) do not apply to our situation. In particular, the equivariant Tian's invariants are $\frac{1}{2}$ for our manifolds (see [Gu6]).

In [Gu2], we considered the scalar curvature equation instead of the Ricci curvature equation in [KS1], and we found that the calculation is simpler. However, the calculation of the scalar curvature is more difficult in the present situation. Therefore, we will deal with the general Kähler classes on $M_{n}$ 's in [Gu6] and those on $N_{n}$ 's in [Gu7] later.

In the third section, we show some special properties of those Kähler metrics on our manifolds, which are some of reasons that our work can be done. We will also try to generalize this calculation to more general Kähler manifolds in [Gu6].

One might also generalize our results to some smooth Hilbert schemes and hence some moduli spaces of holomorphic vector bundles over Kähler-Einstein manifolds.

After we finish our work, we were told about the results of Dancer and Wang [DW] and those of Podestà and Spiro [PS]. Their manifolds are in the class of [KS $]^{2}$ (see [DW Condition 1.12, Proposition 1.15] and [PS Remark 1.4, Lemma 3.2]) and hence none of them is in the semisimple case we considered.

\footnotetext{
${ }^{2}$ Actually the [KS] class is the projectable class in [PS] including the projectable extra-ordinary cases. Therefore, the [PS] class is strictly smaller than that in [KS]. While the [DW] class is even strictly smaller than that of [PS] which includes also some higher multiplicity ones, e.g., one can produce a projectable example from the real structure of the principal orbit in the Example 3.3 in [PS]. They are generic in the sense of the real structure of the principal orbit. However, we notice that for each real structure of the principal orbit there is at least one manifold in the [KS] class associated to it (see the paragraphs after the proof of the Proposition 1.15 in [DW]). Therefore, it seems that their manifolds might not be generic in complex geometry, e.g., in the sense of [Ah] and [HS]. Our examples here is in the extra-ordinary situation and is not projectable, although they are projectable outside $p_{0}$ (see [PS]), hence they are not dealed with in both [DW] and [PS].
} 
In the way of producing Kähler-Einstein metrics on compact manifolds, we also obtain some Kähler-Einstein metrics on open manifolds and manifolds with boundaries. We also discuss this topic in the last section. It seems to us, however, in our situation these cases are easier than the compact case.

2. Some General Results. For any Kähler almost homogeneous manifold $M$, the Albanese map $M \rightarrow A(M)$ is an equivariant holomorphic fiber bundle over $A(M)$ such that each fiber is a simply connected projective almost homogeneous manifold. Let $F$ be the fiber, then the subgroup $A u t^{F}(M)$ of $A u t(M)$ which fixes $F$ is a linear algebraic group. We call $A u t^{F}(M)$ the algebraic part of the automorphism group.

LEMMA 1. If $M$ is a Kähler almost homogeneous manifold of cohomogeneity one with one end and a nonsemisimple algebraic part of the automorphism group, then the Albanese fibration is a product.

Proof. After blowing up, we might assume that the closed orbit is a hypersurface. Only in the case 2) in the Proposition 5.1 of [HS], the statement might not be true, which basically comes from the situation in Corollary 4.7 with $A=$ $N_{A u t^{F}(M)}\left(H^{0}\right) / H=\mathbf{Z}_{2}$. But in the case that $A u t^{F}(M)$ is not semisimple, the Theorem 1 in [Ah p.54] then implies that $A=1$, which is a contradiction.

THEOREM 1. If $M$ is a Kähler almost homogeneous manifold of cohomogeneity one with nonsemisimple algebraic part of the automorphism group, then $M$ is a completion of a $\mathbf{C}^{*}$ bundle over a compact Kähler homogeneous manifold. In particular, every Kähler class has an extremal metric (resp. quasi-Einstein metric).

Proof. If $M$ is an almost homogeneous space with two ends, we apply Theorem 3.2 in [HS p.771]. If $M$ has one end, we apply Lemma 1 and the Theorem 1 in [Ah p.54].

REMARK. For the metrics which are invariant under a maximal connected compact subgroup $K$ of the automorphism group, one can define a modified Mabuchi functional

$$
M\left(\omega_{1}, \omega_{2}\right)=-\int_{a}^{b} \int_{X} \dot{\varphi}_{t}(R-H R-\phi) \omega_{t}^{n} d t,
$$

where $\phi$ is the function corresponding to the extremal vector field $E$ in [FM] (actually we found this functional after reviewing $[\mathrm{FM}]$ in 1995). A local minimal of this functional is achieved by an extremal metric and

$$
M\left(\omega_{1}, g^{*} \omega_{2}\right)=M\left(\omega_{1}, \omega_{2}\right)
$$

for any $g \in C_{K^{\mathbf{C}}}(K)$, where $C_{K^{\mathbf{C}}}(K)$ is the centralizer of $K$ in $K^{\mathbf{C}}$ (see [Gu5] for details and an application to the uniqueness of extremal metrics on smooth toric varieties). One can prove that this functional is well defined as in [ $\mathrm{Mb}$ p.579-580] as following: Let $\psi(s, t)=s \varphi_{t}$, then this problem is reduced to the proof of the closeness of $\Phi(s, t)=\int_{X} \frac{\partial \psi}{\partial s} \phi_{s, t} \omega_{s, t}^{n} d s+\int_{X} \frac{\partial \psi}{\partial t} \phi_{s, t} \omega_{s, t}^{n} d t$. Arguing as in [FM] we see that $\phi_{s, t}=\phi+\frac{1}{2} s\left(d \phi, d \varphi_{t}\right)$ (here we notice that $\phi$ is a real function). Then

$$
\begin{aligned}
d \Phi= & \frac{1}{2} s \int_{X}\left(\varphi_{t}\left(\left(d \phi_{s, t}, d \dot{\varphi}_{t}\right)_{s, t}+\phi_{s, t} \Delta_{s, t}^{d} \dot{\varphi}_{t}\right)\right. \\
& \left.-\dot{\varphi}_{t}\left(\left(d \phi_{s, t}, d \varphi_{t}\right)_{s, t}+\phi_{s, t} \Delta_{s, t}^{d} \varphi_{t}\right)\right) \omega_{s, t}^{n} d t \wedge d s=0 .
\end{aligned}
$$

One might expect that the existence of an extremal metric is equivalent almost to the existence of a lower bound of this functional. The gradient flow of this functional is 
exactly the Calabi flow. And the derivative of this functional along this flow is the negative of the modified Calabi functional

$$
\int_{X} R^{2} \omega^{n}-(H R)^{2} \int_{X} \omega^{n}-F(E),
$$

which is always nonnegative if $\omega$ is invariant under the action of a maximal connected compact subgroup of the automorphism group, and only be zero when $\omega$ is an extremal metric (this can be proved easily with $L^{2}$ decomposition of the real functions as in [Hw2], but in general it seems that the proof there is not correct since one confronts a complex decomposition). Applying Calabi's calculation in [Cl1] to our flow we obtain that the second derivative of the functional is

$$
2 \int_{X} R\left(\dot{\varphi}_{t, \alpha \beta}\right)^{\alpha \beta} \omega_{t}^{n}=2\left(R, \alpha \beta,\left(\dot{\varphi}_{t}\right),_{\alpha \beta}\right)=2\left\|\dot{\varphi}_{t}, \alpha \beta\right\|^{2}>0 .
$$

In other words, the Calabi functional is also decreasing under this flow.

LEMMA 2. Let $V$ be a holomorphic vector field such that the corresponding $(0,1)$ form is harmonic, then the Futaki Invariant $F(V)$ is zero.

Proof. $F(V)=\int_{X} V(f) \omega^{n}=\int_{X} d f(V) \omega^{n}=\left(d f, i_{V} \omega\right)=0$.

THEOREM 2. If $M$ is an almost homogeneous manifold of cohomogeneity one of semsimple type, then the Futaki Invariants are zero.

Proof. We notice that the vanishness of Futaki Invariant is the same up to a finite covering. Therefore, we only consider the first case in the Proposition 5.1 of [HS], where $M$ is a product of a torus and a projective almost homogeneous manifold. The holomorphic vector fields that come from the torus actions have harmonic corresponding $(0,1)$ forms. Hence, by Lemma 2 the corresponding Futaki Invariants are zero. Moreover, the algebraic part of the automorphism group is semisimple. Therefore, the corresponding Futaki Invariants are zero. We get the Theorem.

3. Calculation of the metrics. To calculate the Kähler metrics $\omega$ on $M_{n}$, we consider the pushdown of the metrics by the map $p: M_{n} \rightarrow P_{n}=\mathbf{C} P^{n} \times \mathbf{C} P^{n}$. Then

$$
p_{*} \omega=a \omega_{1}+b \omega_{2}+\partial \bar{\partial} F
$$

where $\omega_{i}(i=1,2)$ are the standard metrics on the first and the second copy of the $\mathbf{C} P^{n}$ 's and $F$ is a function with some singularities on the diagonal.

Now the automorphism group of $M_{n}$ is $P S L(n+1, \mathbf{C})$, and the maximal connected compact subgroup $K=P S U(n+1)$ has real hypersurface orbits. All the $K$ invariant functions are functions of $\theta=\frac{|(z, w)|^{2}}{|z w|^{2}}$ where $z$ and $w$ are the homogeneous coordinates of the $\mathbf{C} P^{n}$ 's. If the metrics are $K$ invariant, then $F$ is a function of $\theta$.

Recall that $\omega_{1}=\partial \bar{\partial} \log |z|^{2}$ and $\omega_{2}=\partial \bar{\partial} \log |w|^{2}$. We also have that $\partial \bar{\partial} F=$ $\partial\left(F^{\prime} \bar{\partial} \theta\right)=\partial\left(\theta F^{\prime} \bar{\partial} \log \theta\right)$. If we let $f=\theta F^{\prime}$, then $f(0)=0$ and

$$
p_{*} \omega=a \omega_{1}+b \omega_{2}+\theta f^{\prime} \partial \log \theta \wedge \bar{\partial} \log \theta+f \partial \bar{\partial} \log \theta
$$

By the symmetric group we only need to calculate the metrics at points with $z_{0}=1, w_{0}=1$ and $z_{i}=w_{j}=0$ if $i \neq 0,1, j \neq 0$. By calculation we obtain:

$$
\begin{aligned}
p_{*} \omega & =(a-f(\theta)) \partial \bar{\partial} \log |z|^{2}+(b-f(\theta)) \partial \bar{\partial} \log |w|^{2} \\
& +f(\theta)\left(\partial_{z} \partial_{\bar{w}}+\partial_{w} \partial_{\bar{z}}\right) \log \theta+\theta f^{\prime}(\theta) \partial \log \theta \wedge \bar{\partial} \log \theta
\end{aligned}
$$




$$
\begin{aligned}
& =(a-f(\theta))\left(\frac{d z \wedge d \bar{z}}{|z|^{2}}-\frac{\left|z_{1}\right|^{2} d z_{1} \wedge d \bar{z}_{1}}{|z|^{4}}\right)+(b-f(\theta)) \frac{d w \wedge d \bar{w}}{|w|^{2}} \\
& +f(\theta)\left(\partial_{z}\left(\frac{z d \bar{w}}{(z, w)}\right)+\partial_{w}\left(\frac{w d \bar{z}}{(w, z)}\right)\right) \\
& +\theta f^{\prime}(\theta)\left(-\frac{\bar{z}_{1} d z_{1}}{|z|^{2}}+\frac{\bar{z}_{1} d w_{1}}{(w, z)}\right) \wedge\left(-\frac{z_{1} d \bar{z}_{1}}{|z|^{2}}+\frac{z_{1} d \bar{w}_{1}}{(z, w)}\right) \\
& =(a-f(\theta))\left(\frac{d z_{1} \wedge d \bar{z}_{1}}{|z|^{4}}+\sum_{i>1} \frac{d z_{i} \wedge d \bar{z}_{i}}{|z|^{2}}\right)+(b-f(\theta)) d w \wedge d \bar{w} \\
& +f(\theta)(d z \wedge d \bar{w}+d w \wedge d \bar{z}) \\
& +\theta f^{\prime}(\theta)\left|z_{1}\right|^{2}\left(\frac{d z_{1}}{|z|^{2}}-d w_{1}\right) \wedge\left(\frac{d \bar{z}_{1}}{|z|^{2}}-d \bar{w}_{1}\right) .
\end{aligned}
$$

We observe that the complex 2-dimensional subspaces $V_{i}$ generated by $\frac{\partial}{\partial z_{i}}, \frac{\partial}{\partial w_{i}}$ are orthogonal to each other for different $i$ with $1 \leq i \leq n$. If we regard the tangent space as the complex vector space generated by the vector fields corresponding to the elements of the Lie algebra of $K$, then the semisimple part of the centralizer of the isotropy group has these $V_{i}$ 's as invariant subspaces of the tangent space. To calculate the volume form, we only need to calculate the determinant $\tau_{i}$ for each $V_{i}$ and compare them with the corresponding items on the standard Kähler-Einstein metric on $\mathbf{C} P^{n} \times \mathbf{C} P^{n}$.

We notice that $\tau_{i}, i \geq 2$ are the same with value:

$$
\left|\begin{array}{cc}
\frac{a-f(\theta)}{|z|^{2}} & f(\theta) \\
f(\theta) & b-f(\theta)
\end{array}\right|=\frac{1}{|z|^{2}}(a-f(\theta))(b-f(\theta))-f^{2}(\theta) .
$$

If $\omega$ comes from the pullback of the standard metric, then $a=b=n+1$ and $\tau_{i}^{0}=$ $\frac{(n+1)^{2}}{|z w|^{2}}$ for $i>1$. So $\frac{\tau_{i}}{\tau_{i}^{0}}=\frac{|z w|^{2}}{(n+1)^{2}}\left(\frac{1}{|z|^{2}}(a-f(\theta))(b-f(\theta))-f^{2}(\theta)\right)$ must be a function of $\theta$, we have

$$
\tau_{i}=\frac{1}{|z w|^{2}}\left((a-f(\theta))(b-f(\theta))-\theta^{-1} f^{2}(\theta)\right)=\frac{1}{|z w|^{2}} A
$$

with $i>1$.

For $i=1$ we have:

$$
\begin{aligned}
& \tau_{1}=\left|\begin{array}{cc}
\frac{a-f(\theta)+\left|z_{1}\right|^{2} \theta f^{\prime}(\theta)}{|z|^{4}} & \frac{f(\theta)|z|^{2}-\left|z_{1}\right|^{2} \theta f^{\prime}(\theta)}{|z|^{2}} \\
\frac{f(\theta)|z|^{2}-\left|z_{1}\right|^{2} \theta f^{\prime}(\theta)}{|z|^{2}} & b-f(\theta)+\theta f^{\prime}(\theta)\left|z_{1}\right|^{2}
\end{array}\right| \\
& =\frac{1}{|z|^{4}}\left(\left(a-f(\theta)+(1-\theta) f^{\prime}(\theta)\right)\left(b-f(\theta)+(1-\theta) f^{\prime}(\theta)\right)\right. \\
& \left.-\left(\theta^{-1} f(\theta)-(1-\theta) f^{\prime}(\theta)\right)^{2}\right) .
\end{aligned}
$$

In the same way, we observe that $\tau_{1}^{0}=\frac{(n+1)^{2}}{|z w|^{4}}$ and hence

$$
\begin{aligned}
\tau_{1} & =\frac{1}{|z w|^{4}}\left(\left(a-f(\theta)+(1-\theta) f^{\prime}(\theta)\right)\left(b-f(\theta)+(1-\theta) f^{\prime}(\theta)\right)\right. \\
& \left.-\left(\theta^{-1} f(\theta)-(1-\theta) f^{\prime}(\theta)\right)^{2}\right) \\
& =\frac{1}{|z w|^{4}} B .
\end{aligned}
$$


We have the following theorem:

THEOREM 3. The volume form is

$$
\frac{1}{|z w|^{2 n+2}} A^{n-1} B d z_{1} \wedge d \bar{z}_{1} \wedge \cdots \wedge d z_{n} \wedge d \bar{z}_{n} \wedge d w_{1} \wedge d \bar{w}_{1} \wedge \cdots \wedge d w_{n} \wedge d \bar{w}_{n}
$$

Now we try to describe the conditions for $f(\theta)$ such that the 2-form defined by $f(\theta)$ is a Kähler form at any point outside the diagonal. We have the following:

LemMa 3. $(A(1-\theta))^{\prime}=-B$.

Proof. $A^{\prime}=\theta^{-2} f^{2}(\theta)-f^{\prime}(\theta)\left(a+b-2 f(\theta)+2 \theta^{-1} f(\theta)\right)$. Therefore, we have

$$
\begin{aligned}
& (A(1-\theta))^{\prime}=A^{\prime}(1-\theta)-A \\
& =\theta^{-2} f^{2}(\theta)-(a-f(\theta))(b-f(\theta)) \\
& -(1-\theta) f^{\prime}(\theta)\left(a+b-2 f(\theta)+2 \theta^{-1} f(\theta)\right) \\
& =-B .
\end{aligned}
$$

We let $C=A(1-\theta)$ and

$$
d V=d z_{1} \wedge d \bar{z}_{1} \wedge \cdots \wedge d z_{n} \wedge d \bar{z}_{n} \wedge d w_{1} \wedge d \bar{w}_{1} \wedge \cdots \wedge d w_{n} \wedge d \bar{w}_{n}
$$

then the volume form is $\frac{C^{n-1} B d V}{|z w|^{2 n+2}(1-\theta)^{n-1}}$. We will see more geometrical meaning of this formula in the next section.

If $\omega$ is positive, then $\tau_{1}>0$. That is, $B>0$. Lemma 3 says that $A(1-\theta)$ is decreasing. We observe that $A$ is positive if and only if $\lim _{\theta \rightarrow 1} A(1-\theta) \geq 0$. Let $\theta=0$, we observe that $a, b>0$ since $\omega$ is positive on $V_{i}$ with $i>1$. On the other hand, if $a, b>0$ and $A>0$, then $\omega$ is positive on $V_{i}$ with $i>1$ at $\theta=0$; and $\omega$ is always positive on $V_{i}(i>1)$ by the continuity (otherwise $\omega$ has zero direction on some $V_{i}(i>1)$, but this contradicts to $\left.A>0\right)$. In the same way, we observe that $\omega$ is positive on $V_{1}$ if and only if $a+f^{\prime}(0), b+f^{\prime}(0)>0$ and $B>0$. We have:

Theorem 4. $\omega$ is a Kähler metric outside the diagonal if and only if (1) $B>0$, (2) $\lim _{\theta \rightarrow 1} C \geq 0$ and $\min \left(a, b, a+f^{\prime}(0), b+f^{\prime}(0)\right) \geq 0$.

4. Determine the singularity of the Kähler potentials. In this section, we will consider how the Kähler metric $\omega$ in Theorem 4 extend to a metric on the diagonal. As in the last section, we only consider the points at which $z_{0}=w_{0}=1$ and $z_{i}=w_{j}=0$ for $i>1, j>0$.

We like to see how the metric on $M_{n}$ comes down to $P_{n}$ near the diagonal. To do this we change the coordinate a little bit. Let $z_{i}^{\prime}=z_{i}-w_{i}$ and $w_{i}^{\prime}=z_{i}+w_{i}$. Then the diagonal is described by $z_{i}^{\prime}=0$. The blow-up effect does not affect $w_{i}^{\prime}$. The blow-up effect can be described by $p:\left(z_{i}^{\prime},\left[u_{i}\right], w_{i}^{\prime}\right) \rightarrow\left(z_{i}^{\prime}, w_{i}^{\prime}\right)$ with $z_{i}^{\prime} u_{j}=z_{j}^{\prime} u_{i}$. At our considered points we have $z_{1}^{\prime}=z_{1}-w_{1}=z_{1}, w_{1}^{\prime}=z_{1}+w_{1}=z_{1}$ and $z_{i}^{\prime}=w_{i}^{\prime}=0$ for $i>1$. They are on the coordinate chart with $u_{1}=1$, that is, $z_{1}^{\prime} u_{i}=z_{i}^{\prime}$ and have coordinates $\left(z_{1}^{\prime}, u_{2}, \cdots, u_{n}, w_{1}^{\prime}, \cdots, w_{n}^{\prime}\right)$.

Therefore, the metric can be written as:

$$
\begin{aligned}
& \omega=a_{11} d z_{1}^{\prime} \wedge d \bar{z}_{1}^{\prime}+a_{1 i} d z_{1}^{\prime} \wedge d \bar{u}_{i}+\bar{a}_{1 i} d u_{i} \wedge d \bar{z}_{1}^{\prime} \\
& +a_{i j} d u_{i} \wedge d \bar{u}_{j}+b_{1 i} d z_{1}^{\prime} \wedge d \bar{w}_{i}^{\prime}+\bar{b}_{1 i} d w_{i}^{\prime} \wedge d \bar{z}_{1}^{\prime} \\
& +b_{i j} d u_{i} \wedge d \bar{w}_{j}^{\prime}+\bar{b}_{i j} d w_{j}^{\prime} \wedge d \bar{u}_{i}+c_{i j} d w_{i}^{\prime} \wedge d \bar{w}_{j}^{\prime}
\end{aligned}
$$


Now we have $u_{i}=\frac{z_{i}^{\prime}}{z_{1}^{\prime}}$, therefore,

$$
d u_{i}=\frac{d z_{i}^{\prime}}{z_{1}^{\prime}}-\frac{z_{i}^{\prime} d z_{1}^{\prime}}{\left(z_{1}^{\prime}\right)^{2}}=\frac{d z_{i}^{\prime}}{z_{1}^{\prime}} .
$$

Applying this into the formula of $\omega$, we find that the coefficient of the $d z_{1} \wedge d \bar{z}_{1}$ term is

$$
a_{11}+b_{11}+\bar{b}_{11}+c_{11}
$$

and the coefficient of the $d z_{2} \wedge d \bar{z}_{2}$ term is

$$
a_{22}\left|z_{1}\right|^{-2}+b_{22} z_{1}^{-1}+\bar{b}_{22} \bar{z}_{1}^{-1}+c_{22} \text {. }
$$

By comparing with our formula for $p_{*} \omega$ we observe that $f(\theta)$ has a single pole at $\theta=1$, i.e., $f(\theta)(1-\theta)$ is a finite function on $[0,1]$.

In the same way, we have $a_{i j}=b_{i j}=c_{i j}=0$ for $i \neq j$, and hence $\lim _{\theta \rightarrow 1} f(\theta)(1-$ $\theta)<0, \lim _{\theta \rightarrow 1} B, C>0$. By calculation we get:

THEOREM 5. Let $\omega$ be a Kähler metric as in Theorem 4. Then it is a metric on the diagonal if and only if $\lim _{\theta \rightarrow 1} f(\theta)(1-\theta)<0, \lim _{\theta \rightarrow 1} B, C>0$.

5. Determine the Equations. In this section, we will concentrate on the case in which $\omega$ is in the Ricci class of $M_{n}$. From Theorem 3, we observe that in this case $a=b=n+1$. Also by Theorem 3, we obtain that the Ricci curvature is

$$
\begin{aligned}
& \operatorname{Ric} \omega=-\partial \bar{\partial} \log \frac{C^{n-1} B}{|z w|^{2 n+2}(1-\theta)^{n-1}} \\
& =\partial \bar{\partial}\left((n+1) \log |z|^{2}+(n+1) \log |w|^{2}\right. \\
& \left.-\log C^{n-1} B+(n-1) \log (1-\theta)\right) .
\end{aligned}
$$

We have that the principal part of $f(\theta)=\theta F^{\prime}(\theta)$ at $\theta=1$ is $-\frac{n-1}{1-\theta}$. Hence, we obtain $\lim _{\theta \rightarrow 1} f(\theta)(1-\theta)=-(n-1)$.

Moreover, we have Kähler-Einstein equation:

$$
F(\theta)=-\log C^{n-1} B+(n-1) \log (1-\theta) .
$$

This equation is equivalent to

$$
F^{\prime}(\theta)=-(n-1) \frac{C^{\prime}}{C}-\frac{B^{\prime}}{B}-\frac{n-1}{1-\theta}=-(n-1) \frac{C^{\prime}}{C}-\frac{C^{\prime \prime}}{C^{\prime}}-\frac{n-1}{1-\theta},
$$

here we apply $C^{\prime}=-B$ in Lemma 3 .

To simplify the equation, we let

$$
V=\theta\left(n+1+\frac{1-\theta}{\theta} f(\theta)\right)^{2} .
$$

Then

$$
\begin{aligned}
& F^{\prime}(\theta)=\frac{f(\theta)}{\theta} \\
& =\frac{1}{1-\theta} \frac{(1-\theta) f(\theta)}{\theta} \\
& =\frac{1}{1-\theta}\left( \pm\left(\frac{V}{\theta}\right)^{\frac{1}{2}}-(n+1)\right),
\end{aligned}
$$


where we must have + sign in the last formula by $\lim _{\theta \rightarrow 1}\left(n+1+\frac{1-\theta}{\theta} f(\theta)\right)=2>0$ and the continuity. We also have

$$
\begin{aligned}
& C=(1-\theta)\left((n+1)^{2}-2(n+1) f(\theta)-f^{2}(\theta) \frac{1-\theta}{\theta}\right) \\
& =(n+1)^{2}-\left(\theta(n+1)^{2}+2(n+1)(1-\theta) f(\theta)+f^{2}(\theta) \frac{(1-\theta)^{2}}{\theta}\right) \\
& =(n+1)^{2}-V .
\end{aligned}
$$

The equation becomes

$$
\frac{\left(\frac{V}{\theta}\right)^{\frac{1}{2}}-2}{1-\theta}=(n-1) \frac{V^{\prime}}{(n+1)^{2}-V}-\frac{V^{\prime \prime}}{V^{\prime}} .
$$

Next we notice that $V^{\prime}=B$. That is, $V$ is increasing and $V(0)=0, V(1)=$ $1(n+1-(n-1))^{2}=4$. We let $U=\frac{V}{4}$. Then the equation becomes

$$
2 \frac{\left(\frac{U}{\theta}\right)^{\frac{1}{2}}-1}{1-\theta}=\frac{4(n-1) U^{\prime}}{(n+1)^{2}-4 U}-\frac{U^{\prime \prime}}{U^{\prime}}
$$

and $U$ is a monotone map from $[0,1]$ onto itself.

THEOREM 6. If there is a solution $U \leq 1$ of (3) with $U(0)=0$ and $U(1)=1$. Then there is a Kähler-Einstein metric on $\bar{M}_{n}$.

Proof. Suppose we have a solution, then by the left side of (3) we must have $U \geq 0$. Now we first want to prove that $U$ is increasing. By letting $U=v^{2}$ and applying the Theorem 10 in [BR p.195] to $v$ in our situation, we observe that if we assume that $U \neq 0, \frac{(n+1)^{2}}{4} ; \theta \neq 0,1$ then the solution is analytic. Since $U \leq 1$ we obtain that if $U>0$ at point $\theta \neq 0,1$ then $U$ is increasing. This is true because if $U^{\prime}=0$ at $\theta$, then $U^{\prime \prime}$ has a zero at $\theta$ of no lower mutiplicity than $U^{\prime}$, a contradiction. Now we want to prove that $U>0$ if $\theta>0$. We notice that by $U(1)=1, U$ can not be always zero, the argument above shows that at some $a<1 U^{\prime}(a)>0$. Then by using $0 \leq U \leq 1$ and (3) we have

$$
-2 \frac{1}{1-\theta} \leq-\left(\log \left((n+1)^{2}-4 U\right)^{n-1} U^{\prime}\right)^{\prime} \leq 2 \frac{\left(\frac{1}{\theta}\right)^{\frac{1}{2}}-1}{1-\theta} .
$$

And by integration we get

$$
\left(\frac{1+\theta^{\frac{1}{2}}}{1+a^{\frac{1}{2}}}\right)^{4} \leq \frac{U^{\prime}(a)}{U^{\prime}(\theta)}\left(\frac{(n+1)^{2}-4 U(a)}{(n+1)^{2}-4 U(\theta)}\right)^{n-1} \leq\left(\frac{1-\theta}{1-a}\right)^{2},
$$

with $0 \leq \theta \leq a<1$. We have $U^{\prime}(0)>0$. That is, $U$ increase always. Now the left side of above inequality still holds when we let $a=1$, we get $U^{\prime}(1)>0$, that is, $B>0$ always.

Second, we let

$$
n+1+\frac{1-\theta}{\theta} f(\theta)=\left(\frac{4 U}{\theta}\right)^{\frac{1}{2}},
$$

then $f(\theta)$ is well defined on $[0,1)$ and $f(0)=0, n+1+f^{\prime}(0)=\left(4 U^{\prime}(0)\right)^{\frac{1}{2}}>$ $0, \lim _{\theta \rightarrow 1}(1-\theta) f(\theta)=2-(n+1)=-(n-1)$. We also have $C(1)=(n+1)^{2}-4>0$. Comparing with the conditions in both Theorems 4 and 5 we obtain the KählerEinstein metric. 
6. The Existence outside the diagonal. Now we consider the equation

$$
U^{\prime \prime}=\frac{4(n-1)\left(U^{\prime}\right)^{2}}{(n+1)^{2}-4 U}-2\left(\frac{\left(\frac{U}{\theta}\right)^{\frac{1}{2}}-1}{1-\theta}\right) U^{\prime} .
$$

Obiviously it has solution $U \equiv a$ on $[a, 1-b]$ with small positive numbers $a, b$. Suppose we already have a solution with $U(a)=a, U(1-b)=c \leq 1$, we want to see that for some $c^{\prime}$ close to and larger than $c$ we have a solution $U(a)=a, U(1-b)=c^{\prime}$. By the continuity of the initial value problem (see [BR p.177 Theorem 5]), if we change $U^{\prime}(a)$ a little larger we shall still get a solution on $[a, 1-b]$. If $U(1-b)>c$, we are done. Otherwise, $U(1-b) \leq c$, hence it must always $\geq a$ and $\leq 1$. Thus, we have the openness of the set $I$ of the initial $U^{\prime}(a)$ 's such that the equation has solutions on $[a, b]$. By (5), we also obtain the closeness of $I$. Therefore, for any large initial $U^{\prime}(a)$, we have a solution on $[a, b]$ with $U \leq 1$, this will lead to a contradiction to (5). Hence, the set of $c^{\prime}$ with solutions is an open set. Now by (5) again, we observe that the set of $c^{\prime}$ is also closed under any number which is close and larger than 1 . Therefore, we can let $c=1$ (This corresponds to a Kähler-Einstein metric on $a \leq \theta \leq 1-b$. In fact, we can have a 2 parameter family of Kähler-Einstein metrics on $0 \leq \overline{U(a)} \leq U(1-b))$. We can also let $a \rightarrow 0$, and obtain:

THEOREM 7. For any small positive number $b$, we have a solution with $U(0)=$ $0, U(1-b)=1$. This corresponds to a Kähler-Einstein metric on the manifold with boundary $\theta \leq 1-b$.

We actually can let $c$ be any number which is nonnegative and smaller than $\left(\frac{n+1}{2}\right)^{2}$. Therefore, we actually obtain a family of Kähler-Einstein metrics on $\theta \leq 1-b$.

7. The Global Existence. In this section we will try to extend our method of finding solutions to the diagonal. We want to see what will happen if we let $b \rightarrow 0$.

Now we notice that the right side of (5) still holds, thus the upper bound of $U^{\prime}$ of the smaller $\theta$ is bounded by the upper bound of $U^{\prime}$ of the larger ones. We will try to bound the $U^{\prime}$ for the $\theta$ 's which are close to 1 .

If $U^{\prime}$ is unbounded. We try to bound $U^{\prime}(1-\theta)$. To do this we let $e^{t}=\frac{1}{1-\theta}$. Let $\theta_{t}$ be the $t$ derivative of $\theta$. Then by differentiation, we have $e^{t}=\frac{\theta_{t}}{(1-\theta)^{2}}=\theta_{t} e^{2 t}$. That is, $\theta_{t}=e^{-t}$. Let $U_{t}$ be the $t$ derivative of $U$, then $U_{t}=U^{\prime} \theta_{t}=U^{\prime} e^{-t}=U^{\prime}(1-\theta)$ and $U^{\prime \prime}=\left(U_{t} e^{t}\right)^{\prime}=U_{t t} e^{2 t}+U_{t} e^{2 t}$, where $U_{t t}$ being the second $t$ derivative of $U$. The equation becomes

$$
2\left(\left(\frac{U}{\theta}\right)^{\frac{1}{2}}-1\right) U_{t}=\frac{4(n-1) U_{t}^{2}}{(n+1)^{2}-4 U}-U_{t t}-U_{t} .
$$

That is,

$$
U_{t t}=\frac{4(n-1) U_{t}^{2}}{(n+1)^{2}-4 U}-\left(2\left(\frac{U}{\theta}\right)^{\frac{1}{2}}-1\right) U_{t} .
$$

Let $U_{i}$ be a series of solutions corresponding to $b_{i} \rightarrow 0$ and $t_{i}$ be the point of $t$ such that $1>U_{i}\left(t_{i}\right)=a>\frac{4}{9}$. Then

$$
\left(\log U_{i, t}\left((n+1)^{2}-4 U_{i}\right)^{n-1}\right)_{t}=-\left(2\left(\frac{U_{i}}{\theta}\right)^{\frac{1}{2}}-1\right)<-\frac{1}{3}
$$

for $t \geq t_{i}$. For any $U$ we define

$$
V=U_{t}\left((n+1)^{2}-4 U\right)^{n-1} .
$$


We notice that $V_{i}=U_{i, t}\left((n+1)^{2}-4 U_{i}\right)^{n-1}$ is decreasing with $t \geq t_{i}$ and $V_{i} \leq$ $e^{-\frac{1}{3}\left(t-t_{i}\right)} V_{i}\left(t_{i}\right)$. We have $U_{i, t} \leq C_{i} U_{i, t}\left(t_{i}\right) e^{-\frac{1}{3}\left(t-t_{i}\right)}$ with $C_{i}$ being bounded both from above and below by some positive numbers which do not depend on $U_{i}$. Then $1-$ $a=\int_{t_{i}}^{t\left(1-b_{i}\right)} U_{i, t} d t<C_{i} U_{i, t}\left(t_{i}\right) \int_{t_{i}}^{+\infty} e^{-\frac{1}{3}\left(t-t_{i}\right)} d t=3 C_{i} U_{i, t}\left(t_{i}\right)$. Therefore, $U_{i, t}\left(t_{i}\right)$ is bounded from blow by a positive number which does not depend on $U_{i}$.

If no subsequence of $t_{i}$ turns to $+\infty$, then a subsequence of $t_{i}$ turn to a finite number $t_{0}$. By the left side of (5), we see that $U_{i}^{\prime}\left(\theta\left(t_{0}\right)\right)$ must be bounded from above, otherwise $U_{i}^{\prime}(\theta(t))$ will be bounded from below by a very large number such that $U_{i}$ will be bigger than 1 before $\theta$ reaching the point 1 . That is, there is a subsequence of $U_{i}$ converging to a solution $U$ of our equation with $U(1) \geq a>\frac{4}{9}$.

We will see that no subsequence of $t_{i}$ turns to $+\infty$. Therefore, by choosing a subsequence we can assume that $t_{i}$ turns to a finite number $t_{0}$ and the $U$ is exactly what we need.

If there is a subsequence of $t_{i}$ turns to $+\infty$. From the equation we observe that if $\frac{4(n-1) U_{i, t}}{(n+1)^{2}-4 U_{i}}>2\left(\frac{1}{\theta\left(t_{i}\right)}\right)^{\frac{1}{2}}-1$, we have that $U_{i, t}$ is increasing. This can not happen. Therefore, $U_{i, t}\left(t_{i}\right)$ is bounded from above. Thus, $\tilde{U}_{i}(t)=U_{i}\left(t+t_{i}\right)$ converges to a nonconstant function $\tilde{U}$, since for each $t \geq 0, V_{i}$ defined by (7) is decreasing and $\tilde{U}_{i, t}$ is bounded; and for each $t<0,-2<\left(\log V_{i}\right)_{t}<1$ when $i$ big enough, that is, $\tilde{U}_{i, t}$ is also bounded as well as $U_{i, t}\left(t_{i}\right)$ is bounded from below by a positive number. Then $\tilde{U}$ satisfy the equation

$$
\left(\log x^{\prime}\left((n+1)^{2}-4 x\right)^{n-1}\right)^{\prime}=-2 x^{\frac{1}{2}}+1
$$

on $(-\infty,+\infty)$. Therefore,

$$
\left(x^{\prime}\left((n+1)^{2}-4 x\right)^{n-1}\right)^{\prime}=\left(-2 x^{\frac{1}{2}}+1\right)\left((n+1)^{2}-4 x\right)^{n-1} x^{\prime} .
$$

We let $u=x^{\prime}\left((n+1)^{2}-4 x\right)^{n-1}$. By integration, we obtain

$$
u=\int_{x(-\infty)}^{x}\left(-2 x^{\frac{1}{2}}+1\right)\left((n+1)^{2}-4 x\right)^{n-1} d x
$$

since $u(-\infty)=0\left(x^{\prime}(-\infty)=0\right)$. Then, by $u(+\infty)=0$ we must have $\int_{x(-\infty)}^{x(+\infty)} f_{n}(x) d x=0$ with $f_{n}(x)=\left(-2 x^{\frac{1}{2}}+1\right)\left((n+1)^{2}-4 x\right)^{n-1}$. Now if we choose ${ }^{3} a$ close enough to 1 and prove that $\int_{0}^{1} f_{n}(x) d x<0$, then

$$
\int_{x(-\infty)}^{x(+\infty)} f_{n}(x) d x<\int_{0}^{x(+\infty)} f_{n}(x) d x<0
$$

when $x(+\infty)$ is close enough to 1 and $x(-\infty)<\frac{1}{4}$. Since $\int_{x(-\infty)}^{x(+\infty)} f_{n}(x) d x<0$ if $x(-\infty) \geq \frac{1}{4}$, we will have a contradiction.

We prove here that $\int_{0}^{1} f_{n}(x) d x<0$. We have

$$
\int_{0}^{1} f_{n}(x) d x
$$

\footnotetext{
${ }^{3}$ This can be proved to be unnecessary. For example, if $b=x(+\infty) \neq 1$, there is a sequence $t_{i}^{\prime} \rightarrow \infty$ such that $\tilde{U}\left(t_{i}^{\prime}\right) \rightarrow b$, that is, $U_{i}\left(t_{i}+t_{i}^{\prime}\right) \rightarrow b$. By our method above, we can use $t_{i}+t_{i}^{\prime}$ instead of $t_{i}$ and obtain a function $\tilde{U}_{1}$. We observe that $\tilde{U}_{1}(-\infty) \geq U_{i}\left(t_{i}\right)=a>\frac{4}{9}>\frac{1}{4}$. Again, a contradiction.
} 


$$
\begin{aligned}
& =\int_{0}^{\frac{1}{4}} f_{n}(x) d x+\int_{\frac{1}{4}}^{1} f_{n}(x) d x \\
& <\int_{0}^{\frac{1}{4}}\left(-2 x^{\frac{1}{2}}+1\right)(n+1)^{2(n-1)} d x+\int_{\frac{1}{4}}^{1}\left(-2 x^{\frac{1}{2}}+1\right)\left((n+1)^{2}-4\right)^{n-1} d x \\
& =\frac{1}{12}(n+1)^{2(n-1)}-\frac{5}{12}\left((n+1)^{2}-4\right)^{n-1} \\
& =\frac{((n+3)(n-1))^{n-1}}{12}\left(\left(1+\frac{4}{(n+3)(n-1)}\right)^{\frac{(n-1)(n+3)}{4} \frac{4}{n+3}}-5\right) \\
& <\frac{((n+3)(n-1))^{n-1}}{12}\left(e^{\frac{4}{n+3}}-5\right) \\
& <0 .
\end{aligned}
$$

By choosing $a$ close enough to 1 , we have $U(1)=1$ as desired.

Now we want to prove that $\lim _{\theta \rightarrow 1} U^{\prime}$ exists and is finite. Let $V=U^{\prime}=U_{t} e^{t}$, then $V_{t}=U_{t t} e^{t}+U_{t} e^{t}=\frac{4(n-1) U_{t}^{2}}{(n+1)^{2}-4 U} e^{t}+2\left(1-\left(\frac{U}{\theta}\right)^{\frac{1}{2}}\right) V$. As in the proof of the existence of $U$, for a $t_{0}$ with $U\left(t_{0}\right)=a^{2}$ such that $1>b=a^{2}-\frac{1}{2}>0$, we have $U_{t}<M e^{-2 b\left(t-t_{0}\right)}$ for $t \geq t_{0}$ with a constant $M$. In particular, $\frac{U_{t}}{e^{-b t}} \rightarrow 0$. Therefore, $\lim _{t \rightarrow+\infty} \frac{U-\theta}{e^{-b t}}=\lim _{t \rightarrow+\infty} \frac{U_{t}-e^{-t}}{-b e^{-b t}}=0$. Thus, $V_{t}<A V e^{-b t}+B V e^{-b t}=C V e^{-b t}$ with some constants $A, B, C=A+B$. That is, $\log \frac{V(t)}{V\left(t_{1}\right)}<\int_{t_{1}}^{+\infty} C e^{-b t} d t$ is bounded from above with any $t>t_{1}>t_{0}$. By choosing special sequence of $t_{1}$ we can get a Cauchy squence of $\log V\left(t_{i}\right)$. That is, $\log V\left(t_{i}\right)$ converges to a finite number, and $V\left(t_{i}\right)$ converges. By choosing other convergent sequence we get same limit. Thus, $U^{\prime}(1)=\lim _{b \rightarrow 0} \frac{U(1-b)-1}{(1-b)-1}=\lim _{\theta \rightarrow 1} U^{\prime}(\theta)$ exists and is finite. By using (3), we have that $U^{\prime \prime}$ is finite on $(0,1)$. And in the same way as above, we have that $U^{\prime \prime}(0)$ and $U^{\prime \prime}(1)$ exists and is finite.

We finally have:

THEOREM 8. There is an unique Kähler-Einstein metric in the Ricci class of $M_{n}$ up to the automorphism of $M_{n}$. $[\mathrm{BM}]$.

Proof. The existence follows from Theorem 6 and the uniqueness follows from

If in Theorem 7 we have $c$ instead of 1 , with $\frac{(n+1)^{2}}{4}>U(1-b)=c>\frac{1}{4}$, then the same method as in the proof of Theorem 8 can also apply to obtain a KählerEinstein metric on $\theta<1$ with $U(1)=c$. We denote these solutions by $U^{c}$ 's. From (5), we see that $\left(U^{c}\right)^{\prime}(1)=\left(U^{c}\right)^{\prime \prime}(1)=0$ for $c<1$ and $\left(U^{c}\right)^{\prime}(1)=\left(U^{c}\right)^{\prime \prime}(1)=+\infty$ for $c>1$. Therefore, we have that when $c>1$, we can extend the metric to the diagonal. But these metrics degenerate at the diagonal. For $c<1$, we can not extend the metrics to the diagonal, hence we have Kähler-Einstein metrics with singularities on the diagonal. We can also apply our arguments for any boundary condition $0 \leq$ $U(a)=c \leq U(1)=c^{\prime}<\frac{(n+1)^{2}}{4}$ and obtain Kähler-Einstein metrics on $a \leq \theta<1$. We have:

THEOREM 9. There is a family of Kähler-Einstein metrics with two parameters on $a \leq \theta<1$.

One might also consider the solution for $0 \leq U(a)=c<U(1-b)=\frac{(n+1)^{2}}{4}$. By a formula which is similar to (5), we obtain $U^{\prime}(1-b)=U^{\prime \prime}(1-b)=+\infty$ in this case. The existence can be proved by a similar argument as above with exchanging $U$ and $\theta$. 
Using a similar argument as in the last section, we can also give an alternative proof. We move $U^{\prime}(a)$ from a very big number to the smaller ones. For any given number $b$, we can obtain a similar formula $(5)^{\prime}$ of (5) which comes from a similar formula of (4) obtained by using $0<U<\frac{(n+1)^{2}}{4}$ instead of $0<U<1$. To save spaces, we do not write out the formula $(5)^{\prime}$ here, but leave it to the readers.

We can choose $U^{\prime}(0)$ big enough such that $U$ pass $\frac{(n+1)^{2}}{4}$ at $\theta=d$ before $\theta$ reaching $1-b$, i.e., $d<1-b$. Then we move $U^{\prime}(0)$ to the smaller ones. Applying $(5)^{\prime}$ to the derivative of $\theta$ to $U$, that is, $\theta_{U}=\left(U^{\prime}\right)^{-1}$, we obtain the continuity of $\theta_{U}$ over $\left[c, \frac{(n+1)^{2}}{4}\right]$, and hence the continuity of $d^{\prime}=\theta\left(\frac{(n+1)^{2}}{4}\right)$. By $(5)^{\prime}$, we have the closeness of the set of $d^{\prime}$ 's. If $d^{\prime}$ 's do not reach $1-b$, we can move $U^{\prime}(0)$ close to 0 . But (5) implies that if $U^{\prime}(0)$ is small enough $\theta$ will pass $1-b$ before $U$ get to 1 , a contradiction. That is, $d^{\prime}$ can be $1-b$. We have following:

THEOREM 10. There is a family of Kähler-Einstein metrics with two parameters on $0 \leq a \leq \theta<1-b$. And there is at least a subfamily of them with one parameter corresponding to $U(1-b)=\frac{(n+1)^{2}}{4}$, hence has singularities on $\theta=1-b$.

Acknowledgement. We thank the Department of Mathematics of Princeton University and NSF as well as others for their supports which make this work possible. We also thank Professors J. Mather and P. Yang for discussions. We thank Professors Lin, C. S. and Xu, X. W. for pointing out two simpler arguments in page 14 .

The first author (Guan) also like to take this opportunity to thank Professors S. Kobayashi and Peter Li for their interests and supports with [Gu2,3].

We also thank Chiu-Chu Liu for pointing out a serious mistake and several defects in a previous version.

\section{REFERENCES}

[Ah] D. AKHIEZE, Equivariant completions of homogeneous algebraic varieties by homogeneous divisors, Ann. Glob. Analysis and Geometry, 1 (1983), pp. 49-78.

[BM] S. BANDO AND T. MABUCHI, Uniqueness of Einstein Kähler metrics modulo connected group actions, in Algebraic Geometry, Sendai 1985, Adv. Stud. Pure Math. 10, 1987, pp. 1140.

[BR] G. BIRKhoff AND G. Rota, Ordinary Differential Equations, Fourth Edition, John Wiley \& Sons, 1989.

[Ch] J. СНEAH, Cellular decompositions for nested Hilbert schemes of points, Pacific J. of Math., 183 (1998), pp. 39-90.

[Cl1] E. CALABI, Extremal Kähler metrics, in Seminars on Differential Geometry, Annals of Math. Studies, Princeton University Press, 1982, pp. 259-290.

[Cl2] E. CALABI, Extremal Kähler metrics II, in Differential Geometry and Complex Analysis, Springer-Verlag, 1985, pp. 95-114.

[DW] A. DANCER AND M. WANG, Kähler-Einstein metrics of cohomogeneity one and bundle constructions for Einstein Hermitian metrics, preprint.

[FM] A. FUTAKI AND T. MABUCHI, Bilinear forms and extremal Kähler vector fields associated with Kähler classes, Math. Ann., 301 (1995), pp. 199-210.

[FMS] A. FUTAKI, T. MABUChI, AND Y. SAKANE, Einstein-Kähler metrics with positive Ricci curvature, in Recent Topics in Complex Geometry, Adv. Studies in Pure Math. 18-I, 1989, pp. 1-73.

[Gu1] Z. Guan, On Certain Complex Manifolds, Dissertation, University of California, at Berkeley, Spring 1993.

[Gu2] Z. GUAN, Existence of extremal metrices on almost homogeneous spaces with two ends, Transaction of AMS, 347 (1995), pp. 2255-2262.

[Gu3] Z. GUAN, Quasi-Einstein metrics, International J. of Math., 6 (1995), pp. 371-379.

[Gu4] D. GUAN, Examples of holomorphic symplectic manifolds which admit no Kähler structure II, Invent. Math., 121 (1995), pp. 135-145. 
[Gu5] D. GuAN, On Modified Mabuchi functional and Mabuchi Moduli space of Kähler metrics on toric bundles, Math. Research Letters, 6 (1999), pp. 547-555.

[Gu6] D. GUAN, Existence of extrimal metrics on almost homogeneous manifolds of cohomogeneity one-II, in preprint.

[Gu7] D. GUAN, Existence of extremal metrics on almost homogeneous manifolds of cohomogeneity one-III, in preprint.

[HS] A. HUCKLEBERRY AND D. SNow, Almost-homogeneous Kähler manifolds with hypersurface orbits, Osaka J. Math., 19 (1982), pp. 763-786.

[Hw1] A. HWANG, On existence of Kähler metrics with constant scalar curvature, Osaka J. Math., 31 (1994), pp. 561-591.

[Hw2] A. HwANG, On the Calabi energy of extremal Kähler metrics, Internat. J. Math., 6 (1995), pp. 825-830.

[KS1] N. KOISO AND Y. SAKANE, Non-homogeneous Kähler-Einstein metrics on compact complex manifolds, in Lecture Notes in Math. 1201, 1986, pp. 165-179.

[KS2] N. KoISO AND Y. SAKANE, Non-homogeneous Kähler-Einstein metrics on compact complex manifolds II, Osaka J. Math., 25 (1988), pp. 933-959.

[Mb] T. MABUCHI, K-energy maps integrating Futaki invariants, Töhuku Math. Journ., 38 (1986), pp. 575-593.

[Nd1] A. NADEL, Multiplier ideal sheaves and Kähler-Einstein metrics of positive scalar curvature, Ann. of Math., 132:2 (1990), pp. 549-596.

[Nd2] A. NADEL, The behavior of multiplier ideal sheaves under morphisms, in Complex Analysis (Wuppertal 1991), Aspects of Math. E17, 1991, pp. 205-222.

[PS] F. PODESTÀ AND A..SPIRO, Kähler manifolds with large isometry groups, preprint.

[Siu] Y. T. SiU, The existence of Kähler-Einstein metrics on manifolds with positive anticanonical line bundle and a suitable finite symmetric group, Ann. of Math., 127:2 (1988), pp. 585627.

[Sk] Y. SAKANE, Examples of compact Kähler-Einstein manifolds with positive Ricci curvatures, Osaka J. Math., 23 (1986), pp. 585-617.

[Ti] G. TIAN, On Kähler-Einstein metrics on certain Kähler manifolds with $\mathbf{C}_{1}(M)>0$, Invent. Math., 89 (1987), pp. 225-246. 
